UDC 519.872, 519.217

PACS 07.05.Tp, 02.60.Pn, 02.70.Bf

DOI: $10.22363 / 2658-4670-2020-28-3-205-215$

\title{
On the rate of convergence for a class of Markovian queues with group services
}

\author{
Anastasia L. Kryukova \\ Vologda State University \\ 15, Lenina St., Vologda, 160000, Russian Federation \\ (received: July 12, 2020; accepted: September 14, 2020)
}

There are many queuing systems that accept single arrivals, accumulate them and service only as a group. Examples of such systems exist in various areas of human life, from traffic of transport to processing requests on a computer network. Therefore, our study is actual. In this paper some class of finite Markovian queueing models with single arrivals and group services are studied. We considered the forward Kolmogorov system for corresponding class of Markov chains. The method of obtaining bounds of convergence on the rate via the notion of the logarithmic norm of a linear operator function is not applicable here. This approach gives sharp bounds for the situation of essentially non-negative matrix of the corresponding system, but in our case it does not hold. Here we use the method of 'differential inequalities' to obtaining bounds on the rate of convergence to the limiting characteristics for the class of finite Markovian queueing models. We obtain bounds on the rate of convergence and compute the limiting characteristics for a specific non-stationary model too. Note the results can be successfully applied for modeling complex biological systems with possible single births and deaths of a group of particles.

Key words and phrases: queuing system, Markovian queues, forward Kolmogorov system, rate of convergence, limiting characteristics

\section{Introduction}

Consider a Markovian queueing model on the finite state space $\{0,1, \ldots, N\}$ with single arrivals and group services, see the first motivation in [1] and more recent studies in [2], [3].

Let $X(t)$ be the corresponding queue-length process for any $t \geqslant 0$. Denote by $p_{i j}(s, t)=P\{X(t)=j \mid X(s)=i\}, i, j \geqslant 0,0 \leqslant s \leqslant t$ the transition probabilities of $X(t)$ and by $p_{i}(t)=P\{X(t)=i\}$ - the probability that the Markov chain $X(t)$ is in state $i$ at time $t$. Let $\mathbf{p}(t)=\left(p_{0}(t), p_{1}(t), \ldots, p_{N}(t)\right)^{T}$ be the vector of state probabilities at the moment $t$.

(C) Kryukova A. L., 2020 
The probabilistic dynamics of the process $X(t)$ is described by the forward Kolmogorov system

$$
\frac{d \mathbf{x}}{d t}=A(t) \mathbf{x}
$$

where $A(t)=Q^{T}(t)$ is the transposed intensity matrix. All column sums of this matrix are zeros for any $t \geqslant 0$, and $A(t)$ is essentially nonnegative (i.e. all its off-diagonal elements are nonnegative for any $t \geqslant 0$ ), and all 'intensity functions' $a_{i j}(t)$ are analytical in $t$.

We suppose that $a_{i j}(t)=0$ for $i>j-1$, all rates service do not depend on the size of a queue, i.e. $a_{i, i+k}(t)=b_{k}(t)$ for $k \geqslant 1$, arrival rates $a_{i, i-1}(t)=$ $\lambda_{i}(t)$. The process $X(t)$ belongs to class (III), see [3]. The matrix $A(t)$ for $X(t)$ has the following structure:

$$
A(t)=\left(\begin{array}{ccccccc}
a_{00}(t) & b_{1}(t) & b_{2}(t) & b_{3}(t) & \ldots & b_{N-1}(t) & b_{N}(t) \\
\lambda_{1}(t) & a_{11}(t) & b_{1}(t) & b_{2}(t) & \ldots & b_{N-2}(t) & b_{N-1}(t) \\
0 & \lambda_{2}(t) & a_{22}(t) & b_{1}(t) & \ldots & b_{N-3}(t) & b_{N-2}(t) \\
0 & 0 & \lambda_{3}(t) & a_{33}(t) & \ddots & b_{N-4}(t) & b_{N-3}(t) \\
\vdots & \vdots & \vdots & \ddots & \ddots & \ddots & \vdots \\
0 & 0 & 0 & 0 & \ddots & a_{N-1 N-1}(t) & b_{1}(t) \\
0 & 0 & 0 & 0 & \ldots & \lambda_{N-1}(t) & a_{N N}(t)
\end{array}\right) .
$$

\section{Stationary Markovian queueing model}

In this paper we consider a subclass of the class (III) satisfying additional suppositions $b_{i}(t)=0,1 \leqslant i \leqslant N-1, b_{N}(t)=b(t)$ and $\lambda_{i}(t)=\lambda(t)$ for any $i$, $t \geqslant 0$.

The difficulty of studying this model is due to the fact that it is not possible to apply the most convenient method of the logarithmic norm for it, see [3].

Now we get the following expression for the transposed intensity matrix:

$$
A(t)=\left(\begin{array}{cccccc}
a_{00}(t) & 0 & 0 & \cdots & 0 & b(t) \\
\lambda(t) & a_{11}(t) & 0 & \cdots & 0 & 0 \\
0 & \lambda(t) & a_{22}(t) & \cdots & 0 & 0 \\
\vdots & \vdots & \vdots & \ddots & \vdots & \vdots \\
0 & 0 & 0 & \cdots & a_{N-1 N-1}(t) & 0 \\
0 & 0 & 0 & \cdots & \lambda(t) & a_{N N}(t)
\end{array}\right)
$$

We perform the following system transformations. Since $p_{0}(t)=1-$ $\sum_{i \geqslant 1} p_{i}(t)$, one can rewrite the system (1) as

$$
\frac{d \mathbf{z}}{d t}=B(t) \mathbf{z}+\mathbf{f}(t)
$$


where $\mathbf{f}(t)=(\lambda(t), 0, \ldots, 0)^{T}, \mathbf{z}=\left(p_{1}(t), p_{2}(t), \ldots, p_{N}(t)\right)^{T}$,

$$
B(t)=\left(\begin{array}{ccccccc}
a_{11}-\lambda & -\lambda & -\lambda & -\lambda & \ldots & -\lambda & -\lambda \\
\lambda & a_{22} & 0 & 0 & \ldots & 0 & 0 \\
0 & \lambda & a_{33} & 0 & \ldots & 0 & 0 \\
0 & 0 & \lambda & a_{44} & \ddots & 0 & 0 \\
\vdots & \vdots & \vdots & \ddots & \ddots & \ddots & \vdots \\
0 & 0 & 0 & 0 & \ddots & a_{N-1 N-1} & 0 \\
0 & 0 & 0 & 0 & \ldots & \lambda & a_{N N}
\end{array}\right) .
$$

All bounds on the rate of convergence to the limiting regime for $X(t)$ correspond to the same bounds of the solutions of system

$$
\frac{d \mathbf{y}}{d t}=B(t) \mathbf{y}(t)
$$

Denote by $T$ upper triangular matrix

$$
T=\left(\begin{array}{ccccc}
1 & 1 & 1 & \cdots & 1 \\
0 & 1 & 1 & \cdots & 1 \\
0 & 0 & 1 & \cdots & 1 \\
\vdots & \vdots & \vdots & \ddots & \vdots \\
0 & 0 & 0 & \cdots & 1
\end{array}\right)
$$

Let $\mathbf{u}(t)=T \mathbf{y}(t)$, then

$$
\frac{d \mathbf{u}}{d t}=B^{*}(t) \mathbf{u}(t)
$$

where

$$
B^{*}(t)=T B(t) T^{-1}=\left(\begin{array}{cccccc}
-\lambda & 0 & 0 & \cdots & 0 & -b \\
\lambda & -\lambda & 0 & \cdots & 0 & -b \\
0 & \lambda & -\lambda & \cdots & 0 & -b \\
0 & 0 & \lambda & \ddots & 0 & -b \\
\vdots & \vdots & \vdots & \ddots & \ddots & \vdots \\
0 & 0 & 0 & \cdots & \lambda & -(\lambda+b)
\end{array}\right)
$$

Let us remark that the matrix $B^{*}(t)$ is not essentially non-negative. This means that the method of the logarithmic norm is inconvenient to apply (it gives poor results). That's why we use the method of 'differential inequalities', which was described in [4]-[6].

Let $\mathrm{D}=\operatorname{diag}\left(d_{1}, d_{2}, \ldots d_{N}\right)$ be a diagonal matrix and $d_{i}, i=1, \ldots, N$ be nonzero numbers. By $\mathbf{w}(t)$ denote a product $\mathrm{Du}(t)$, then one can rewrite $(8)$ as following system 


$$
\frac{d \mathbf{w}}{d t}=B^{* *}(t) \mathbf{w}(t)
$$

where

$$
B^{* *}(t)=\mathrm{D} B^{*}(t) \mathrm{D}^{-1}=\left(\begin{array}{ccccccc}
-\lambda & 0 & 0 & 0 & \cdots & 0 & -b \cdot \frac{d_{1}}{d_{N}} \\
\lambda \cdot \frac{d_{2}}{d_{1}} & -\lambda & 0 & 0 & \cdots & 0 & -b \cdot \frac{d_{2}}{d_{N}} \\
0 & \lambda \cdot \frac{d_{3}}{d_{2}} & -\lambda & 0 & \cdots & 0 & -b \cdot \frac{d_{3}}{d_{N}} \\
\vdots & \vdots & \vdots & \vdots & \ddots & \vdots & \vdots \\
0 & 0 & 0 & 0 & \cdots & -\lambda & -b \cdot \frac{d_{N-1}}{d_{N}} \\
0 & 0 & 0 & 0 & \cdots & \lambda \cdot \frac{d_{N}}{d_{N-1}} & -\lambda-b
\end{array}\right) .
$$

By $\mathbf{u}(t)$ denote an arbitrary solution of system (8), then we can consider an interval $\left(t_{1}, t_{2}\right)$ with fixed signs of the coordinates $u_{i}(t)$ and choose the elements of the diagonal matrix such that signs of the entries $d_{i}$ are equal with signs of corresponding coordinates $u_{i}(t)$ of the solution of system (8).

Since any $d_{k} u_{k}(t) \geqslant 0$ on the corresponding time interval, the sum $\sum_{k=1}^{N} d_{k} u_{k}(t)=\|\mathbf{w}\|$ can be considered as the corresponding norm.

Denote $B^{* *}(t)=\left(b_{i j}^{* *}(t)\right)_{i, j=1}^{N}$. Now, if the function $\alpha_{D}(t)$ is such that $\sum_{i=1}^{N} b_{i j}^{* *}(t) \leqslant-\alpha_{D}(t), j=1, \ldots, N$, then the following bound holds:

$$
\frac{d\|\mathbf{w}\|}{d t}=\frac{d\left(\sum_{i=1}^{N} w_{k}\right)}{d t}=\sum_{j=1}^{N} \sum_{i=1}^{N} b_{i j}^{* *}(t) w_{j} \leqslant-\alpha_{D}(t)\|\mathbf{w}\| .
$$

We set $\alpha^{*}=\min \left\{\alpha_{D}(t)\right\}$, where the minimum is taken over all intervals with different combinations of coordinate signs of the solution. Hence for any such combination we have the inequality

$$
\|\mathbf{w}(t)\| \leqslant e^{-\int_{s}^{t} \alpha^{*}(\tau) d \tau}\|\mathbf{w}(s)\| .
$$

If we compared all the norms, then one can obtain the final bound

$$
\|\mathbf{y}(t)\| \leqslant C e^{-\int_{0}^{t} \alpha^{*}(\tau) d \tau}\|\mathbf{y}(0)\|,
$$

where $C$ is a corresponding constant.

In our case (in general, all intensities depend on the time $t$ )

$$
\sum_{i=1}^{N-1} w_{i}^{\prime}=-\lambda \cdot\left(1-\frac{d_{2}}{d_{1}}\right) \cdot w_{1}-\lambda \cdot\left(1-\frac{d_{3}}{d_{2}}\right) \cdot w_{2}-\lambda \cdot\left(1-\frac{d_{4}}{d_{3}}\right) \cdot w_{3}-\cdots-
$$




$$
-\left(\lambda+b \cdot\left(1+\frac{d_{1}}{d_{N}}+\frac{d_{2}}{d_{N}}+\cdots+\frac{d_{N-1}}{d_{N}}\right)\right) \cdot w_{N-1}
$$

1) Let all $u_{1}, \ldots, u_{N}$ be positive. Since $\left(1-\frac{d_{i+1}}{d_{i}}\right)$ must be positive, we have $d_{i}>d_{i+1}$. Suppose $d_{1}:=h, d_{2}:=h^{2}, \ldots, d_{N}:=h^{N}$, then

$$
\begin{aligned}
\sum_{i=1}^{N-1} w_{i}^{\prime}= & -\lambda \cdot\left(1-\frac{d_{2}}{d_{1}}\right) \cdot w_{1}-\lambda \cdot\left(1-\frac{d_{3}}{d_{2}}\right) \cdot w_{2}-\lambda \cdot\left(1-\frac{d_{4}}{d_{3}}\right) \cdot w_{3}-\cdots- \\
& -\left(\lambda+b \cdot\left(1+\frac{d_{1}}{d_{N}}+\frac{d_{2}}{d_{N}}+\cdots+\frac{d_{N-1}}{d_{N}}\right)\right) \cdot w_{N-1}= \\
=-\lambda \cdot(1-h) \cdot w_{1}-\lambda \cdot(1-h) \cdot w_{2}-\lambda \cdot(1-h) \cdot w_{3}-\cdots- & -\left(\lambda+b \cdot\left(1+\frac{1}{h}+\frac{1}{h^{2}}+\cdots+\frac{1}{h^{N-1}}\right)\right) \cdot w_{N-1}
\end{aligned}
$$

and we have $\alpha_{D}=\lambda \cdot(1-h)$.

2) Let all $u_{1}, \ldots, u_{k}$ be positive, and all $u_{k+1}, \ldots, u_{N}$ negative. Similarly $\left|d_{i}\right|>\left|d_{i+1}\right|$. Suppose $d_{1}:=h^{N-k+1}, d_{2}:=h^{N-k+2}, \ldots, d_{k}:=h^{N}, d_{k+1}:=-h$, $d_{k+2}:=-h^{2}, \ldots, d_{N}:=-h^{N-k}$, then

$$
\begin{aligned}
\sum_{i=1}^{N-1} w_{i}^{\prime}= & -\lambda \cdot\left(1-\frac{d_{2}}{d_{1}}\right) \cdot w_{1}-\lambda \cdot\left(1-\frac{d_{3}}{d_{2}}\right) \cdot w_{2}-\lambda \cdot\left(1-\frac{d_{4}}{d_{3}}\right) \cdot w_{3}-\cdots- \\
& -\left(\lambda+b \cdot\left(1+\frac{d_{1}}{d_{N}}+\frac{d_{2}}{d_{N}}+\cdots+\frac{d_{N-1}}{d_{N}}\right)\right) \cdot w_{N-1}= \\
=-\lambda & \cdot(1-h) \cdot w_{1}-\lambda \cdot(1-h) \cdot w_{2}-\lambda \cdot(1-h) \cdot w_{3}-\cdots- \\
& -\lambda \cdot(1-h) \cdot w_{k-1}-\lambda \cdot\left(1+\frac{1}{h^{N-1}}\right) \cdot w_{k}-\cdots- \\
-(\lambda+b \cdot & \left.\left(1-h-h^{2}-\ldots-h^{k}+\frac{1}{h^{N-k-1}}+\frac{1}{h^{N-k-2}}+\cdots+\frac{1}{h}\right)\right) \cdot w_{N}
\end{aligned}
$$

In this case $\alpha_{D}=\lambda \cdot(1-h)$ too.

3) Let all $u_{1}, \ldots, u_{k}$ be positive, and all $u_{k+1}, \ldots, u_{s}$ negative and all $u_{s+1}$, $\ldots, u_{N}$ be positive too. As before $\left|d_{i}\right|>\left|d_{i+1}\right|$. Suppose $d_{1}:=h^{N-k+1}, d_{2}:=$ $h^{N-k+2}, \ldots, d_{k}:=h^{N}, d_{k+1}:=-h^{N-s+1}, d_{k+2}:=-h^{N-s+2}, \ldots, d_{s}:=-h^{N-k}$, $d_{s+1}:=h, d_{s+2}:=h^{2}, \ldots, d_{N}:=-h^{N-s}$.

$$
\begin{aligned}
\sum_{i=1}^{N-1} w_{i}^{\prime}= & -\lambda \cdot\left(1-\frac{d_{2}}{d_{1}}\right) \cdot w_{1}-\lambda \cdot\left(1-\frac{d_{3}}{d_{2}}\right) \cdot w_{2}-\lambda \cdot\left(1-\frac{d_{4}}{d_{3}}\right) \cdot w_{3}-\cdots- \\
& -\left(\lambda+b \cdot\left(1+\frac{d_{1}}{d_{N}}+\frac{d_{2}}{d_{N}}+\cdots+\frac{d_{N-1}}{d_{N}}\right)\right) \cdot w_{N-1}=
\end{aligned}
$$




$$
\begin{gathered}
=-\lambda \cdot(1-h) \cdot w_{1}-\lambda \cdot(1-h) \cdot w_{2}-\lambda \cdot(1-h) \cdot w_{3}-\cdots- \\
-\lambda \cdot(1-h) \cdot w_{k-1}-\lambda \cdot\left(1+\frac{1}{h^{s-1}}\right) \cdot w_{k}-\lambda \cdot(1-h) \cdot w_{k+1}-\cdots-\lambda \cdot(1-h) \cdot w_{s-1}- \\
-\lambda \cdot\left(1+\frac{1}{h^{N-k}}\right) \cdot w_{s}-\lambda \cdot(1-h) \cdot w_{s+1}-\cdots- \\
-\left(\lambda+b\left(1+h^{s-k+1}+\cdots+h^{s}-h-h^{2}-\cdots-h^{s-k}+\frac{1}{h^{N-k-1}}+\cdots+\frac{1}{h}\right)\right) \cdot w_{N}
\end{gathered}
$$

as before $\alpha_{D}=\lambda \cdot(1-h)$.

In the general case, we do the same. Then we have $C=h^{1-N}$ and the following bound

$$
\|y(t)\| \leqslant 2 N h^{1-N} \cdot e^{-\lambda \cdot(1-h) t}\|y(0)\| .
$$

In general non-stationary situation

$$
\|y(t)\| \leqslant 2 N h^{1-N} \cdot e^{-(1-h) \int_{0}^{t} \lambda(\tau) d \tau}\|y(0)\| .
$$

\section{Non-stationary Markovian queueing model}

Here we consider a specific queueing model with 1-periodic intensities: $\lambda(t)=2+\sin (2 \pi t)$ and $b(t)=(2+\cos (2 \pi t))$, then

$$
A(t)=\left(\begin{array}{ccccccc}
a_{00} & 0 & 0 & 0 & \cdots & 0 & 2+\cos (2 \pi t) \\
2+\sin (2 \pi t) & a_{11} & 0 & 0 & \cdots & 0 & 0 \\
0 & 2+\sin (2 \pi t) & a_{22} & 0 & \cdots & 0 & 0 \\
\ddots & \ddots & \ddots & \ddots & \ddots & \ddots & \ddots \\
0 & 0 & 0 & 0 & \cdots & a_{N-1 N-1} & 0 \\
0 & 0 & 0 & 0 & \cdots & 2+\sin (2 \pi t) & a_{N N}
\end{array}\right)
$$

and $B^{* *}(t)=\left(b_{i j}^{* *}(t)\right)_{i, j=1}^{N}$, where $b_{i i}^{* *}(t)=-2-\sin (2 \pi t)$, if $1 \leqslant i \leqslant N-1$; $b_{N N}^{* *}(t)=-4-\sin (2 \pi t)-\cos (2 \pi t), b_{i N}^{* *}(t)=-(2+\cos (2 \pi t)) \cdot \frac{d_{i}}{d_{N}}$, if $1 \leqslant i \leqslant$ $N-1 ; b_{i(i-1)}^{* *}(t)=(2+\sin (2 \pi t)) \cdot \frac{d_{i}}{d_{i-1}}$, if $2 \leqslant i \leqslant N$, all other elements are zero.

Then we have the following bound on the rate of convergence

$$
\|y(t)\| \leqslant 2 N \cdot h^{1-N} \cdot e^{-\int_{0}^{t}(1-h) \lambda(\tau) d \tau}\|y(0)\|,
$$

namely

$$
\|y(t)\| \leqslant 2 N \cdot h^{1-N} \cdot e^{-(1-h) \int_{0}^{t}(2+\sin (2 \pi \tau)) d \tau}\|y(0)\|,
$$

hence

$$
\|\mathbf{y}(t)\| \leqslant 2 N \cdot h^{1-N} \cdot e^{-(1-h) t}\|\mathbf{y}(0)\| .
$$

The right-hand side of estimate (16) decreases rather slowly with increasing $t$. However, this does not mean that the estimate is inaccurate because the real 
rate of convergence is rather slow. For example, let $N=50$ and $h=\frac{1}{2}$. In Figures $1-3$ we can see the state probability of an empty queue $p_{0}(t)$ as low convergence rate function of time $t$.

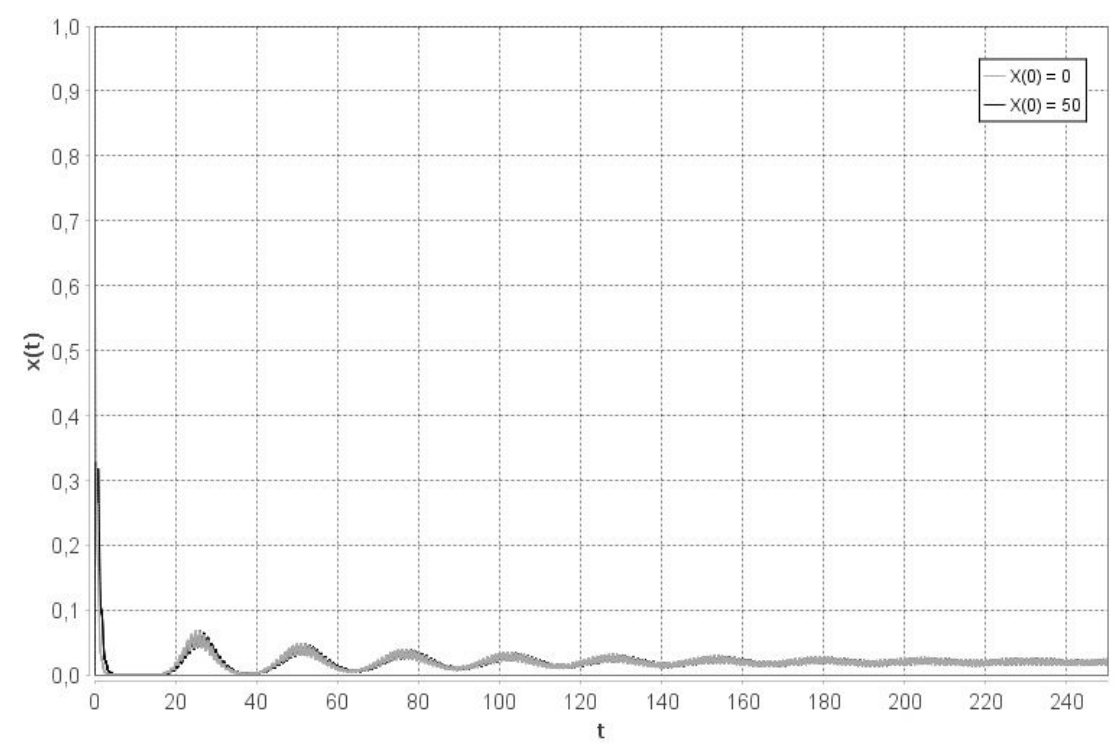

Figure 1. Probability of the empty queue $p_{0}(t)$ for $t \in[0,250]$ with initial conditions $X(0)=50$ (black) and $X(0)=0$ (gray)

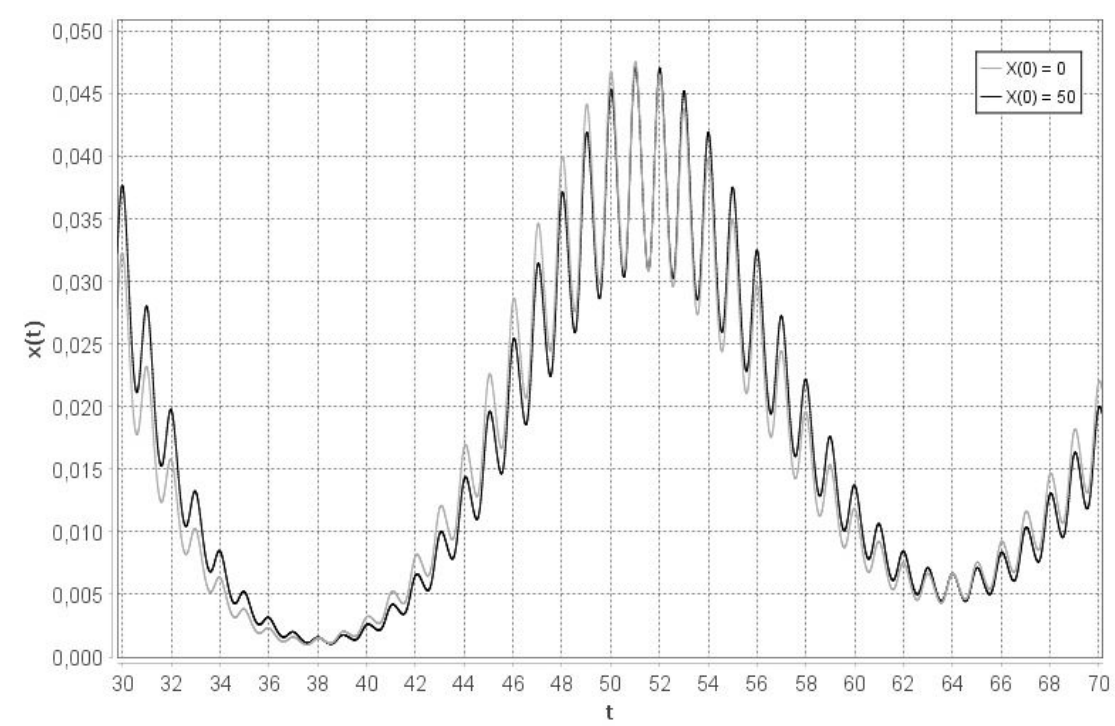

Figure 2. Probability of the empty queue $p_{0}(t)$ for $t \in[30,70]$ with initial conditions $X(0)=50$ (black) and $X(0)=0$ (gray) 


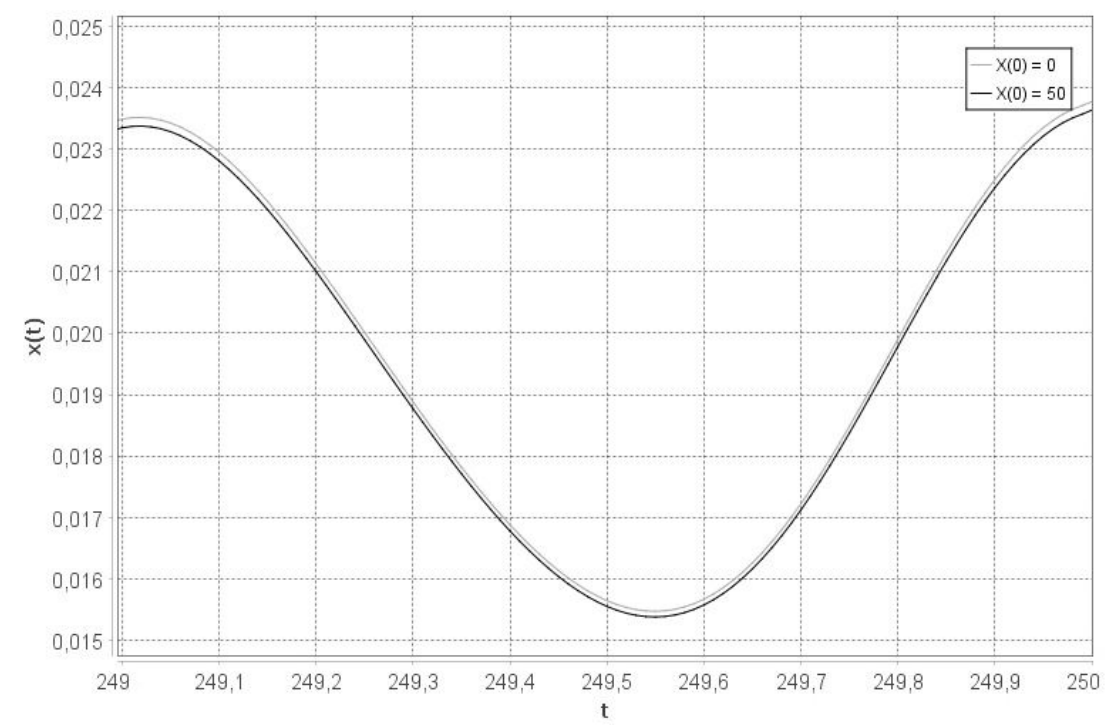

Figure 3. Probability of the empty queue $p_{0}(t)$ for $t \in[249,250]$ with initial conditions $X(0)=50$ (black) and $X(0)=0$ (gray)

\section{Conclusions}

Some new class of finite Markovian queueing models with single arrivals and group services was considered. Bounds on the rate of convergence for these models and computations of the limiting characteristics for a specific non-stationary model were obtained.

The obtained results belong to the theory of queueing systems and can be applied, for example, in medical and biological stochastic systems, which satisfy the adopted assumptions.

For describing possibility of applications of Markovian queues we can refer to [7]-[17], which contains a broad overview and a classification of timedependent queueing systems.

\section{Acknowledgments}

This research was supported by Russian Science Foundation under grant 19-11-00020.

\section{References}

[1] R. Nelson, D. Towsley, and A. N. Tantawi, "Performance analysis of parallel processing systems," IEEE Transactions on Software Engineering, vol. 14, no. 4, pp. 532-540, 1988. DOI: $10.1109 / 32.4676$.

[2] J. Li and L. Zhang, " $M^{X} / M / c$ Queue with catastrophes and statedependent control at idle time," Frontiers of Mathematics in China, vol. 12, no. 6, pp. 1427-1439, 2017. DOI: 10.1007/s11464-017-0674-8. 
[3] A. Zeifman, R. Razumchik, Y. Satin, K. Kiseleva, A. Korotysheva, and V. Korolev, "Bounds on the rate of convergence for one class of inhomogeneous Markovian queueing models with possible batch arrivals and services," International Journal of Applied Mathematics and Computer Science, vol. 28, pp. 141-154, 2018. DOI: 10.2478 /amcs2018-0011.

[4] A. Zeifman, A. Sipin, V. Korolev, G. Shilova, K. Kiseleva, A. Korotysheva, and Y. Satin, "On Sharp Bounds on the Rate of Convergence for Finite Continuous-time Markovian Queueing Models," in Computer Aided Systems Theory - EUROCAST 201\%. EUROCAST 201\%. Lecture Notes in Computer Science, R. Moreno-Díaz, F. Pichler, and A. QuesadaArencibia, Eds., vol. 10672, 2018, pp. 20-28. DOI: 10.1007/978-3-31974727-9_3.

[5] A. Zeifman, Y. Satin, A. Kryukova, R. Razumchik, K. Kiseleva, and G. Shilova, "On the Three Methods for Bounding the Rate of Convergence for some Continuous-time Markov Chains," International Journal of Applied Mathematics and Computer Science, vol. 30, pp. 251-266, 2020. DOI: $10.34768 /$ amcs-2020-0020.

[6] Y. Satin, A. Zeifman, and A. Kryukova, "On the Rate of Convergence and Limiting Characteristics for a Nonstationary Queueing Model," Mathematics, vol. 7, no. 678, pp. 1-11, 2019. DOI: 10.3390/ math7080678.

[7] B. Almasi, J. Roszik, and J. Sztrik, "Homogeneous finite-source retrial queues with server subject to breakdowns and repairs," Mathematical and Computer Modelling, vol. 42, no. 5, pp. 673-682, 2005. DOI: 10. 1016/j.mcm. 2004.02.046.

[8] A. Brugno, C. D'Apice, A. Dudin, and R. Manzo, "Analysis of an $M A P / P H / 1$ queue with flexible group service," International Journal of Applied Mathematics and Computer Science, vol. 27, no. 1, pp. 119131, 2017. DOI: 10.1515/amcs-2017-0009.

[9] A. Di Crescenzo, V. Giorno, B. K. Kumar, and A. Nobile, "A TimeNon-Homogeneous Double-Ended Queue with Failures and Repairs and Its Continuous Approximation," Mathematics, vol. 6, no. 5, p. 81, 2018. DOI: $10.3390 /$ math6050081.

[10] A. N. V. Giorno and S. Spina, "On some time non-homogeneous queueing systems with catastrophes," Applied Mathematics and Computation, vol. 245, pp. 220-234, 2014. DOI: 10.1016/j .amc.2014.07.076.

[11] B. Granovsky and A. Zeifman, "Nonstationary Queues: Estimation of the Rate of Convergence," Queueing Systems, vol. 46, pp. 363-388, 2004. DOI: 10.1023/B:QUES.0000027991.19758.b4.

[12] H. LI, Q. Zhao, and Z. Yang, "Reliability Modeling of Fault Tolerant Control Systems," International Journal of Applied Mathematics and Computer Science, vol. 17, no. 4, pp. 491-504, 2007. DOI: 10.2478/ v10006-007-0041-0.

[13] A. Moiseev and A. Nazarov, "Queueing network $M A P-(G I / \infty) K$ with high-rate arrivals," European Journal of Operational Research, vol. 254, no. 1, pp. 161-168, 2016. DOI: 10.1016/j.ejor.2016.04.011. 
[14] J. Schwarz, G. Selinka, and R. Stolletz, "Performance analysis of timedependent queueing systems: Survey and classification," Omega, vol. 63, pp. 170-189, 2016. DOI: 10.1016/j.omega.2015.10.013.

[15] N. Vvedenskaya, A. Logachov, Y. Suhov, and A. Yambartsev, "A Local Large Deviation Principle for Inhomogeneous Birth-Death Processes," Problems of Information Transmission, vol. 54, no. 3, pp. 263-280, 2018. DOI: $10.1134 / \mathrm{S} 0032946018030067$.

[16] A. Zeifman, S. Leorato, E. Orsingher, Y. Satin, and G. Shilova, "Some universal limits for nonhomogeneous birth and death processes," Queueing Systems, vol. 52, no. 2, pp. 139-151, 2006. DOI: 10.1007/s11134006-4353-9.

[17] A. Zeifman, V. Korolev, Y. Satin, A. Korotysheva, and V. Bening, "Perturbation Bounds and Truncations for a Class of Markovian Queues," Queueing Systems, vol. 76, no. 2, pp. 205-221, 2014. DOI: 10.1007/ s11134-013-9388-0.

For citation:

A.L.Kryukova, On the rate of convergence for a class of Markovian queues with group services, Discrete and Continuous Models and Applied Computational Science 28 (3) (2020) 205-215. DOI: 10.22363/2658-46702020-28-3-205-215.

Information about the authors:

Kryukova, Anastasia L. - Lecturer of Department of Applied Mathematics of Vologda State University (e-mail: kryukovaforstudents@gmail.com, phone: +7(911)513-79-62, ORCID: https://orcid.org/0000-0001-8145-8846) 
УДК $519.872,519.217$

PACS 07.05.Tp, 02.60.Pn, 02.70.Bf

DOI: $10.22363 / 2658-4670-2020-28-3-205-215$

\title{
О скорости сходимости одного класса марковских цепей с групповым обслуживанием требований
}

\author{
А. Л. Крюкова \\ Вологодский государственный университет \\ ул. Ленина, д. 15, Вологда, 160000, Россия
}

Существует множество систем массового обслуживания, которые принимают единичные требования, накапливают их и обслуживают только как группу. Примеры таких систем можно найти в различных областях человеческой жизни - от трафика транспортных перевозок до обработки запросов в компьютерных сетях. Этим обуславливается актуальность нашего исследования. В этой статье изучается некоторый класс конечных марковских моделей массового обслуживания с одиночным прибытием и групповым обслуживанием. Рассмотрена прямая система Колмогорова для соответствующего класса цепей Маркова. Метод определения границ сходимости, основанный на понятии логарифмической нормы, здесь не применим. Такой подход даёт точные оценки для моделей, для которых матрица соответствующей системы существенно неотрицательна, но в нашем случае это не так. Здесь мы использовали новый метод «дифференциальных неравенств» для получение оценки скорости сходимости для этого класса конечных марковских моделей. Кроме того, мы получили оценки скорости сходимости и вычислили предельные характеристики и для соответствующей нестационарной модели. Заметим, что результаты могут быть успешно применены для моделирования сложных биологических систем, в которых возможны рождения новых особей только по одной и гибель групп.

Ключевые слова: система массового обслуживания, марковский процесс, прямая система Колмогорова, скорость сходимости, предельные характеристики 